\title{
Valor económico, nutricional y medicinal de hongos comestibles silvestres
}

\author{
Economic, nutritional and medicinal \\ value of edible wild mushrooms
}

\begin{abstract}
Wild edible mushrooms (WEM) have high economic and culinary value, due to their nutritional and medical properties. They are regarded as functional foods, as well as of the nutritional properties that they have, it has been shown to have beneficial health effects and may be used in the prevention or treatment of diseases. It's therapeutic action is attributed to the bioactive compounds that they have in their fruiting bodies. Although, globally, it has only been studied for $6 \%$ of the fungal diversity. There is a wide range of WEM which can be consumed safely and that can be exploited for the development food products, therefore it is necessary to promote future researches with WEM to reveal more information about their health benefits, because currently available information is still scanty. In this paper the importance of studying the WEM were described, the physical and chemical characteristics and the bioactive compounds of the most popular genus of mushroom in the food area were also described in order to know their nutritional and therapeutic benefits, eliminate false beliefs around them and encourage consumption.
\end{abstract}

Key words: Wild edible mushroom; economy value; nutritional properties; medicinal properties; bioactive compounds.

\section{INTRODUCCIÓN}

El estudio sistemático de los hongos tiene tan sólo 250 años, pero las manifestaciones de este grupo de organismos se conocen desde hace cientos de años (1). Basándose en la diversidad vegetal mundial, reportaron que posiblemente existían entre 1500000 a 2500000 especies de hongos (2). En México se estimó que pueden haber 200000 especies, de las cuales, sólo se conocen 7000 aproximadamente (3).

Los indígenas de las comunidades boscosas tienen un gran conocimiento de los hongos comestibles silvestres (HCS) y se han convertido en un recurso muy estimado para ellos. Son recolectados en la temporada de lluvias identificándolos en base a su forma, color o consistencia; sitio donde crecen y época de desarrollo, donde los pobladores han aprendido a clasificarlos por el conocimiento que han heredado de sus antepasados (4). Los nombres tradicionales con los que son referidas las especies por parte de los pobladores, son fundamentales para interpretar sus criterios de clasificación que permite catalogarlos en sistemas jerárquicos de manera muy similar a las occidentales. Los criterios principales de agrupamiento desprenden los nombres populares (5). Un ejemplo de ello, son los hongos del genero Ramaria, a los cuales nombran como escobitas o escobetas, debido la forma ramificada del
Araceli Cano-Estrada (1)

Leticia Romero-Bautista (2)

(1) Universidad Autónoma del Estado de Hidalgo. Escuela Superior de Tlahuelilpan Área Académica de Enfermería Ex Hacienda de San Servando. Av. Universidad $s / n$. Col Centro. Tlahuelilpan, Hidalgo. México, CP. 42840 (2) Laboratorio de Etnobotánica. Universidad Autónoma del Estado de Hidalgo. Instituto de Ciencias Básicas e Ingeniería Ciudad del Conocimiento Mineral de la Reforma, Hidalgo, CP. 42184, México.

Dirigir la correspondencia a: Araceli Camo Estrada Tel. 7717172000 Ext.5506 edith_cano@uaeh.edu.mx edith_ara@yahoo.com

Este trabajo fue recibido el 10 de Septiembre de 2015 y aceptado para ser publicado el 14 de Enero de 2016. hongo. Otro, son los hongos del género Lactarius que se les conoce popularmente como enchilados, pero a Lactarius indigo se le nombra enchilado azul debido a su coloración (tabla 1). Sin embargo, hace falta desarrollar más investigaciones para la identificación de especies de HCS, pues se estima que sólo se conoce $3.5 \%$.

Los macromicetos más conocido entre la población son aquellos comestibles y cultivables como el champiñón (Agaricusbisporus) y la seta (Pleurotusspp) que se pueden encontrar en los supermecados. Sin embargo, cada vez son más las especies silvestres de hongos comestibles que se cotizan en mercados y mercados ambulantes denominados "tianguis" en México, por lo que cada día los investigadores están más interesados en caracterizar diferentes especies, con el fin de conocer sus propiedades nutricionales y terapéuticas, encontrar técnicas de cultivo adecuadas que permitan en un futuro su comercialización y en el desarrollo de nuevos productos culinarios hechos a base de hongos.

Los HCS son considerados un alimento funcional, ya que se ha demostrado que su consumo impacta de forma positiva a una o más funciones del organismo.

El concepto de alimento funcional fue introducido en Japón a mediados de los años 1980, donde los platillos elabora- 
dos bajo este concepto recibieron el nombre de Alimentos para el Uso Específico de la Salud (FOSHU por sus siglas en inglés) (6). Esta corriente ocasionó que las actuales investigaciones sobre alimentos se enfoquen en la eliminación de sustancias poco favorables para la salud e incrementar el contenido de sustancias con efectos positivos para el ser humano. Las propiedades de los hongos son únicas, representan un reino con características muy diferentes al de las plantas y animales y su potencial como alimento medicinal es muy amplio pero poco difundido entre la población.

El objetivo de este artículo fue revisar los conocimientos en cuanto a la clasificación y propiedades nutricionales que se han obtenido de los géneros más representativos de HCS, así como de la importancia ecológica y comercial que actualmente tiene este tipo de recurso natural.

\section{VALOR ECONÓMICO}

La producción mundial de los hongos cultivados supera los 6.2 millones de toneladas, cuyo valor se aproxima a los 30 billones de dólares. La tasa de incremento de la producción anual es del $11 \%$ y esto se debe a la investigación, confirmación y difusión de sus propiedades medicinales y nutritivas. Por esta razón, se observa un alza en la demanda de productos derivados de hongos comestibles (7).

El volumen de producción de hongos frescos en México se estima en 38708 toneladas anuales, lo que representa alrededor del 59\% del total de la producción en Latinoamérica, siendo este país el 180 productor a nivel mundial (8). Su exportación genera divisas por más de cuatro millones de dólares anuales. Las operaciones comerciales tienen un monto anual aproximado de 150000000 de dólares, generando alrededor de empleos directos e indirectos. La importancia ecológica de esta actividad económica radica en la utilización y reciclaje de más de 386000 toneladas anuales de subproductos agrícolas, agroindustriales y forestales obtenidos del hongo.

Los principales hongos cultivados actualmente es el champiñon (Agaricusbisporus), la seta (Pleurotus spp.) el shiitake (Lentinula edodes) y Volvariella volvácea. El género Agaricus ha sido el hongo más cultivado y consumido en Europa, Norteamérica y México, éste último con una producción de 36500 ton/año. Por su parte, Lentinula edodes es producido en su mayor parte en Japón, Volvariella volvácea en Asia y Pleurotus en Sudamérica y México, con una producción de 2 190 ton/año en este país (8).

Por otra parte, este recurso no es aprovechado en todo su potencial y los beneficios económicos son aún limitados por falta de organización, procesamiento, regulación y conocimiento científico y tecnológico (9). Por lo tanto, el conocimiento ecológico plantea las bases para una explotación sustentable. Los HCS pueden ser consumidos por diferentes larvas de insectos, insectos, caracoles, ardillas, entre otros animales, lo que resulta en una interacción benéfica para el medio ambiente. Sin embargo, con la deforestación que sufren los bosques actualmente, se encuentran fuertemente amenazados. Alrededor de 100000 Km2 de bosques se están perdiendo al año, y como consecuencia se calcula que una cuarta parte de diversidad mundial se perderá en los siguientes 25 años, lo que significa que alrededor de 350000 especies de hongos se habrán extinguido durante este período (3).

\section{VALOR NUTRICIONAL Y MEDICINAL}

Los macromicetos son considerados ingredientes principales de platillos tradicionales y gourmet, así como también excelentes acompañantes de innumerables formas de preparación. Aunque se continúan considerando como una amenaza a las especies venenosas y letales, la realidad es que los episodios de muertes y envenenamientos son pocos y raros comparados con el consumo cotidiano y seguro de las especies silvestres. Algunas sociedades, la publicidad y los estilos culturales siguen sembrando temor al respecto. Esto acontece con mayor frecuencia en los países desarrollados y ha llevado a la creencia generalizada de que el consumo mundial de los HCS se da en pequeña escala y está restringido a áreas en las que su presencia es más abundante.

Los HCS han sido recolectados y consumidos por la gente durante miles de años. China aparece preponderantemente en el registro histórico de este grupo de organismos, quienes han apreciado muchas especies con el pasar de los siglos, no sólo por sus propiedades nutritivas y el sabor sino también por sus propiedades curativas. Hoy en día China es el líder en exportaciones de hongos cultivados.

Registros arqueológicos revelan especies de HCS asociadas con las poblaciones chilenas desde hace 13000 años (10). También fueron recolectados en los bosques en tiempos de la antigua Grecia y de los romanos, siendo apreciados más por personas de alto rango que por la población en general (11).

El uso de hongos en la dieta de los seres humanos ha prevalecido debido a su sabor y olor característico. Sin embargo, en los últimos años el interés por los HCS se ha intensificado, ya que constituyen una fuente importante de nutrientes. Presentan una composición química que los hace atractivos desde el punto de vista nutricional; en general, contienen $90 \%$ de agua y $10 \%$ de materia seca, de los cuales $27-48$ $\%$ son de proteína, aproximadamente $60 \%$ corresponde a carbohidratos, en especial fibras dietéticas (D-glucanas, quitina y sustancias pécticas) y 2-8\% son lípidos (12), entre los cuales destaca el ácido linoléico $(13,14)$. El alto contenido proteico, (15 al 35\% del peso seco), refleja las creencias que los hongos son un sustituto efectivo de la carne, aunque no todos los hongos silvestres contienen gran cantidad de proteínas, su valor nutritivo puede ser comparado con el de muchas especies vegetales. El contenido de minerales en los hongos comestibles varía entre 6 y $11 \%$ según la especie; los que aparecen en mayor cantidad son el calcio, potasio, fósforo, magnesio, zinc y cobre (15). En cuanto al contenido de vitaminas, los hongos comestibles son ricos en riboflavina (B2), niacina (B3) y folatos (B9) (15).

Por otra parte, los macromicetos producen metabolitos secundarios como los compuestos fenólicos, los pigmentos carotenoides y el ergosterol que reducen el riesgo de contraer enfermedades, especialmente cáncer o trastornos cardiovasculares $(16,17)$. Los polifenoles son compuestos químicos que poseen una actividad antioxidante efectiva en los sistemas biológicos (18), actúan también como agentes antiinflamantorios y contra el envejecimiento celular $(19,20)$, e interfieren en la iniciación y progresión de cáncer (21).

Los hongos han trascendido más allá de mitos, cuentos y leyendas; se presentan como un potencial culinario en muchos países, a través de la manifestación de diversos productos y platillos. Los hongos microscópicos por un lado, con la elaboración de quesos, panes y algunas bebidas industrializadas como el vino. Pero los HCS siguen siendo la base en la elaboración de un sinnúmero de platillos, desde los tradicionales hasta los más exóticos (22). A diferencia de otros alimentos, se distinguen por sus variadas formas, aromas, colores, sabores, texturas y tamaños.

Comer HCS se reviste de múltiples aspectos, ya que a diferencia de otros alimentos, se trata de organismos que sólo 
crecen en temporada de lluvias, en determinados ecosistemas y lugares específicos y que además implica un reconocimiento profundo de las especies seguras. A continuación se describen las características morfológicas y nutricionales de algunas de las especies comestibles más cotizadas para el consumo humano.

Agaricus. Es un amplio género de hongos, que pertenecen al orden de los Agaricales y a la familia de los Agaricaceae. Las principales características de las especies que pertenecen a este género son: velo general no diferenciado del revestimiento de píleo. Sombrero frecuentemente convexo, de colores blancos, pálidos y marrones. Láminas libres al pie, de jóvenes cremosas o rosadas y en su madurez cafés a negruzcas; estípite central separable del píleo con un anillo membranoso sencillo o doble, superior o inferior (23).

Agaricus bisporus es la especie más representativa de este género, comúnmente conocido como champiñón y es el más cultivado a nivel mundial. Se estima que en el 2009 se produjeron alrededor de 4000000 de toneladas. En cuanto a su composición química se reporta un contenido de humedad del $91.4 \%$ y por lo tanto $8.6 \%$ de materia seca, de las cuales alrededor del 19\% son proteínas, $23 \%$ fibra (24) y 12 $\%$ minerales cuyo análisis mostró altas cantidades de potasio, fosforo, cobre y hierro (25).

Estudios han revelado que Agaricus bisporus posee actividad antioxidante atribuida a la presencia de aproximadamente $7 \mu \mathrm{g}$ de compuestos fenólicos y alrededor de $3 \mu \mathrm{g}$ de flavonoides (tabla 1), ayudando a prevenir el envejecimiento celular, con la reducción de los efectos de las especies reactivas de oxígeno; además de poseer propiedades antiinflamatorias y ayudar a prevenir cierto tipo de cáncer (18).

Amanita. Es uno de los géneros más fascinantes de hongos silvestres, pertenecen al orden de los Agaricales y son muy cotizados en mercados establecidos y mercados ambulantes, pues se consideran de los favoritos en la gastronomía tradicional, aunque también este género contiene especies de hongos altamente venenosas y alucinógenas, por lo que la mayoría de las investigaciones realizadas se han concentrado en estudiar a este tipo de Amanitas con el fin de caracterizar sus toxinas.

Entre las especies que se consumen se encuentra Amanita caesarea, Amanita fulva, Amanita rubescens (26). Amanita caesarea también conocida como Amanita de los cesares debido a que los romanos, en especial el emperador Julio Cesar, eran muy aficionados a degustarla. Presenta un alto contenido de agua, $(93.8 \%)$ y por lo tanto $6.2 \%$ es de materia seca, la cual contiene un $13 \%$ de proteína, $11.3 \%$ de minerales y un alto contenido de fibra 16.4\% (27) (tabla 2).

Por otra parte, se tiene a las especies más tóxicas como Amanita phaoides, Amanita virosa, Amanita verna y Amanita visporigera; un pequeño trozo del cuerpo fructífero de estos hongos es capaz de ocasionar la muerte. Amanita muscaria es otra especie cuya toxicidad es ocasional y desde el principio del siglo XVII se le da un uso de alucinógeno, así como de insecticida por lo que es común que se le nombre como el hongo de la mosca (28).

Boletus. Pertenece al orden Boletales y a la familia de los boletaceae. Estos hongos forman un grupo de especies morfológicamente variable que presentan un himenóforo poroide, laminar, liso, tubular o dentado (29). En especial, la familia boletaceae se caracteriza por tener un himelío forrado de tubos, fácilmente separables del píleo, que desemboca en poros y cuyo tamaño y color característico depende de la especie (30). Una característica importante para la identificación de estos hongos es la tinción de color azul que sufre el hongo al ser recolectado, debido a la oxidación (30), aunque la intensidad de la coloración puede variar según la especie.

Entre los hongos más cotizados en la gastronomía se encuentra Boletus edulis, ya que está considerado entre los hongos más deliciosos. A este hongo se le conoce popularmente como "panadero bueno" (tabla 1). Se reportan la presencia de compuestos fenólicos (alrededor de $14 \mu \mathrm{g}$ ) y flavonoides (alrededor de $2.5 \mu \mathrm{g}$ ) (18). También se han realizado estudios sobre sus propiedades antimicrobianas, inhibiendo a bacterias como Escherichia Coli y Staphylococcus aureus (31), haciendo que este tipo de hongo además de delicioso sirva para fines terapéuticos (tabla 1). Por otra parte, para su composición química se reporta $90.8 \%$ de humedad y $9.2 \%$ de materia seca (27). Presenta $18.5 \%$ de proteína cruda, $6.9 \%$ de minerales totales y alrededor de $23 \%$ de fibra cruda, (tabla 2).

Algunas otras especies comestibles descritas en la literatura son: Boletus edilus, Boletus aerus, Boletu saestivalis y Boletu ssp.

Lactarius. Pertenece al orden de Russulales y a la familia Russulaceae. Este género se caracteriza por la secreción de látex del cuerpo fructífero cuando es cortado y por su coloración que varía entre azul obscuro y azul pálido. En general, su píleo mide entre 5 y $20 \mathrm{~cm}$ y de forma plana-convexa cuando son jóvenes y cóncavos cuando llegan a la madurez. Crecen en bosques templados y en diversas especies de este género se ha observado la asociación simbiótica a las raíces de los árboles.

Entre las especies que se reportan comestibles se encuentran: Lactarius deliciosus, lactarius indigo, lactarius deterrimus y lactarius sanguifluus. Actualmente, algunas de estas especies han cobrado mayor importancia no sólo por su valor gastronómico sino por sus propiedades farmacéuticas que han revelado estudios recientes (32), donde destacan la actividad antimicrobiana debido a la presencia de compuestos sesquiterpénicos que actúan sobre bacterias como Escherichia coli, Enteroccocus facecium, Bacillus subtilus y Staphylococcus aureus (tabla 1). También se ha reportado actividad anticancerígerna de los compuestos activos de este tipo de hongos (32).

Pleurotus. Este género se encuentra dentro del orden de los Agaricales y de la familia de las Pleurotaceae. Se encuentra dentro de los hongos comestibles más comercializados. En México, el cultivo comercial de los hongos Pleurotus comenzó en el año de 1974 en las zonas boscosas cercanas a la capital. La producción anual estimada se ha incrementado significativamente; para 1991 fue de 356 toneladas, mientras que para el año 2009 fueron 2920 toneladas (33). Actualmente, en la mayoría de las poblaciones son comercializados en fresco o son empaquetados en platos de polietileno dentro de mercados o en tiendas de autoservicio.

Al menos 15 especies de Pleurotus han sido reconocidas y estudiadas mostrando su gran potencial como alimentos funcionales y con utilidad biotecnológica (33). Pleurotus ostreatus es conocido comúnmente como hongo ostra, oreja blanca o simplemente seta $(34,35)$; presenta un píleo en forma de repisa de 4 a $14 \mathrm{~cm}$. De diámetro, color blanquecino o gris. Contiene. Esta especie corresponde a un alimento altamente proteico, alrededor del $17.5 \%$ y contiene altas cantidades de fibra, (23\%), (24). Posee un elevado contenido de tiamina (vitamina B1), rivoflabina (B2), piridoxina (B6) y cobalamina (B12) y es una fuente importante de calcio y fósforo (34). Estudios revelan que este hongo posee alrededor de $7 \mu \mathrm{g}$ de compuestos fenólicos y aproximadamente $0.8 \mu \mathrm{g}$ de flavonoides, importantes para prevenir la oxidación celular (18) (tabla 1).

Por otra parte, Pleurotus spp. contiene $92.4 \%$ de humedad y $7.6 \%$ de materia seca (27). El $7.3 \%$ de la materia seca 
corresponde a minerales totales, $15.4 \%$ a proteínas crudas y $22 \%$ a fibra cruda (tabla 2), además de poseer actividad antibiótica y antiviral (36).

Algunas otras especies comestibles de este género son: Pleurotus pulmonarius, Pleurotus eryngii, Pleurotus djamor.

Ramaria. Pertenece al orden de las Gamphales y a la familia de las Gomphaceas. Es un género poco estudiado, pero con características muy particulares. A diferencia de los hongos antes descritos, los hongos del género Ramaria presentan ramificaciones en su cuerpo fructífero. Esta peculiar característica, aunada al colorido que presentan las diferentes especies de este género, los han bautizado también como hongos de coral (37). La gama de colores que podemos encontrar es amplia, y varían entre amarillo, naranja, rojo y púrpura. Algunos ejemplos de especies comestibles de este género son: Ramaria flava que posee una coloración amarilla, Ramaria

\section{TABLA 1}

Efecto medicinal y compuestos bioactivos de hongos silvestres comestibles.

\begin{tabular}{|c|c|c|c|c|}
\hline Especie de Hongo & Nombre popular & Efecto medicinal & Compuestos bioactivos & Referencia \\
\hline Agaricus bisporus & Champiñón & Antioxidante & $\begin{array}{l}\text { Compuestos fenólicos } \\
\text { Flavonoides } \\
\beta \text { - Carotenos }\end{array}$ & (18) \\
\hline Boletus edulis & $\begin{array}{l}\text { Panadero,Cemita, } \\
\text { Pancita. }\end{array}$ & Antioxidante & $\begin{array}{l}\text { Compuestos fenólicos } \\
\text { Flavonoides } \\
\beta \text { - Carotenos }\end{array}$ & (18) \\
\hline Pleurotus spp. & Seta & $\begin{array}{l}\text { Antiviral } \\
\text { Antibiotica }\end{array}$ & Polisacaridos & $\begin{array}{l}(38) \\
(38)\end{array}$ \\
\hline Pleurotus osteatrus & $\begin{array}{l}\text { Hongo ostra, } \\
\text { oreja blanca. }\end{array}$ & $\begin{array}{l}\text { Antioxidante } \\
\text { Antibiótico } \\
\text { Antibacterial } \\
\text { Antitumoral }\end{array}$ & $\begin{array}{l}\text { Compuestos fenólicos } \\
\text { Flavonoides } \\
\beta \text {-Carotenos } \\
\text { Polisacaridos } \\
\beta \text {-D-Glucano } \\
\text { Glicopeptidos }\end{array}$ & $\begin{array}{l}(38) \\
(38) \\
(38)\end{array}$ \\
\hline Lactarius deliciosus & Enchilado & Antibacterial & sequiterpenos & (32) \\
\hline Lactarius indigo & $\begin{array}{l}\text { Enchilados azules, } \\
\text { Azulejos, } \\
\text { Orejas azules. }\end{array}$ & $\begin{array}{l}\text { Antitumoral } \\
\text { Antiinflamatorios }\end{array}$ & $\begin{array}{l}\text { Extractos orgánicos: } \\
\text { Terpenoides } \\
\text { Polifenoles } \\
\text { Extractos orgánicos: } \\
\text { Terpenoides } \\
\text { Polifenoles }\end{array}$ & (32) \\
\hline Ramaria Botrytis & $\begin{array}{l}\text { Hongo coral } \\
\text { Escobitas. }\end{array}$ & Antioxidante & $\begin{array}{l}\text { Compuestos fenólicos } \\
\text { Tecoferol } \\
\text { Carotenoides } \\
\text { Ácido ascórbico }\end{array}$ & (36) \\
\hline
\end{tabular}

TABLA 2

Propiedades químicas de hongos comestibles silvestres.

\section{Especie de Hongo}

Agaricus bisporus

Amanita caesarea

Boletus edulis

Pleurotus ostreatus

Pleurotus spp.

Ramaria flava
Gramos por cada $100 \mathrm{~g}$ de materia fresca

Humedad Grasa cruda Minerales Proteína cruda

$\begin{array}{ccccc}91.4 & 0.3 & 0.8 & 1.8 & 2.0 \\ 93.8 & \mathrm{nr} & 0.7 & 0.81 & 1.02 \\ 90.8 & 0.5 & 0.6 & 1.7 & 2.1 \\ 92 & 0.4 & 0.9 & 1.6 & \mathrm{nr} \\ 92.4 & \mathrm{nr} & 0.6 & 1.2 & 1.7 \\ 92.7 & \mathrm{nr} & 0.6 & 1.1 & 1.7\end{array}$

$\mathrm{nr}=$ datos no reportados 
araiospora que presenta un color rojo coral, Ramaria sp. con una coloración amarillo pálido y Ramaria botrytis con una coloración rosada. De esta última especie se han estudiado sus propiedades medicinales encontrando que tiene una alta actividad antioxidante y alto contenido de compuestos fenólicos y flavonoides (37). Además posee propiedades antimicrobianas contra bacterias gran positivas como Bacillus subtilus, Bacillus cereus y Staphylococcus aureus (tabla 1).

Para la especie de Ramaria flava se reporta su caracterización química con un contenido de humedad del $92.7 \%$ y por lo tanto $7.3 \%$ de materia seca (27). Contiene $15.4 \%$ de proteínas, $8.5 \%$ de minerales totales y $23 \%$ de fibra cruda (tabla 2 ).

\section{CONCLUSIÓN}

Los HCS representan un recurso natural poco estudiado, pero a pesar de que ello, cada día es más la importancia socio-económica que están logrado. Los estudios acerca de las propiedades terapéuticas y nutricionales de algunas especies de HCS han aumentado el interés por su consumo, pero aún hace falta mayor investigación para llevar a cabo la caracterización química de muchas más especies, así como la difusión de ésta información que permita erradicar los diferentes mitos que giran en torno a su consumo, que incluso han llegado a satanizarlos. En general, los HCS tienen un elevado contenido de agua, proteínas y de fibra, además de un gran poder antioxidante, debido al alto contenido de compuestos fenólicos, como se ha demostrado en cepas de Agaricus, Boletus, Ramaria, Lactarius y Pleurotus. Por otra parte, se ha estudiado el poder antimicrobiano en especies de Boletus (Boletus aestivalis y Boletus edilus), de Ramaria (Ramaria botritys) y varias especias de Lactarios, así como del uso de extractos de Lactarius indigo para prevenir crecimiento de células tumorales. Estos hallazgos sobre las propiedades medicinales de los hongos pueden aprovecharse para el desarrollo de nuevos productos culinarios y de esta manera fomentar su consumo; como se ha realizado con el hongo Gonoderma leccidum, pues actualmente podemos encontrar productos comerciales, como el café, enriquecido con extractos de este hongo, debido a sus a propiedades antiinflamatorias y antitumorales.

Aún son pocos los hongos comestibles que son cultivados y comercializados, uno de ellos es el comúnmente llamado champiñón, cuya producción en México asciende a 40000 toneladas, el shittake (Lectinula edodes), que en Asia tiene un gran valor comercial. Pero, existen muchas especies comestibles silvestres con un valor gastronómico elevado, debido a su sabor, olor y propiedades nutricionales, que no se han comercializado a gran escala debido a la falta de estudios sobre las condiciones de crecimiento de estos hongos, o bien porque algunos de ellos no pueden ser cultivables.

Es importante idear estrategias para el uso responsable de estos hongos, así como dar a conocer las propiedades benéficas para la salud del ser humano y así ofrecer al público formas tradicionales y alternativas para aprovechar de manera responsable las diferentes especies de Hongos comestibles silvestres.

\section{RESUMEN}

Los hongos comestibles silvestres (HCS) tienen un alto valor económico y gastronómico, debido a sus propiedades nutricionales y medicinales. Son considerados como alimentos funcionales, pues además de sus propiedades nutricionales, se ha demostrado efectos benéficos para la salud que pueden ser utilizados en la prevención o tratamiento de enfermedades. Su acción terapéutica es atribuida a los compuestos bioactivos que poseen en sus cuerpos fructíferos. A nivel mundial, sólo se ha estudiado $6 \%$ de la diversidad fúngica; existe una amplia gama de HCS que pueden ser consumidos con seguridad y que pueden ser aprovechados para el desarrollo de productos alimenticios, por lo tanto es necesario impulsar futuras investigaciones que revelen mayor información sobre sus propiedades nutricionales y beneficios para la salud, ya que actualmente la información disponible aun es escasa. En el presente artículo se describe la importancia del estudio de estos vegetales, y se detallan las características físicas, químicas y los principios activos de algunos de los géneros de hongos más populares en el ámbito alimenticio con el fin de dar a conocer sus beneficios nutricionales y terapéuticos, así como de eliminar las falsas creencias entorno a ellos.

Palabras clave: Hongos comestibles silvestres; valor económico; propiedades nutricionales; propiedades medicinales; compuestos bioactivos.

\section{BIBLIOGRAFÍA}

1. Alexopolus CJ, Mims CW and Blackwell M. Introductory Micology. John Wiley and Sons Inc.USA. 1996.

2. Hawksworth DL.The fungal dimension of the biodiversity, significance and conservation. Micol Res. 1991; 95:641-55.

3. Guzmán G. Inventoring the fungi of México. Biod and Conser 1998a; 7:369-384.

4. Romero-Bautista L, Islas-Santillán MA, Pulido-Flores $G$, Valdez-Romero $X$. Recetario de Hongos. Tianguis y Mercados del Estado de Hidalgo. Universidad Autónoma del Estado de Hidalgo, Hidalgo 2013.

5. Guzmán G. Vázquez-Dávila M.A. ed. Los Hongos comestibles, medicinales y sagrados de México. La etnobiología en México: reflexiones y experiencias. Secretaria de Educación Pública/Asociación de Etnobiología en México.,1999, p.145-151.

6. Shimizu T. Newly established regulation in Japan: foods with health claim. Asia Pacific J Clin Nutr. 2002; 11(2):94-6.

7. Boa E. Los Hongos silvestres comestibles: perspectiva global de su uso e importancia para la población. FAO. Roma. 2005, p. 50-62.

8. Martínez-Carrera D, Sabal M, Morales P, Martínez W, Martínez M, Mayett Y. Los Hongos comestibles: propiedades nutricionales, medicinales y su contribución a la alimentación mexicana. Escuela de postgraduados. México, 2004.

9. Garibay-Orijel R. Martínez-Ramos M. y Cifuentes J. Disponibilidad de esporomas de hongos comestibles en los bosques de pino-encino de Ixtlán de Juárez. Rev Mexicana Biodiversidad 2009; 80:521-34.

10. Rojas C y Mansur E. Ecuador: informaciones generales sobre los productos no madereros en Ecuador. En Memoria, consulta de expertos sobre productos forestales no madereros para América Latina y el Caribe,Serie Forestal \#1. Santiago. FAO oficina Regional para América Latina y el Caribe, 1995 p 208-23.

11. Buller AHR.The fungus lores of the Greeks and Romans. Transactions of the British Mycol Soc. 1914;5:21-66.

12. Sánchez C. Modern aspects of mushroom culture technology. Applled Microbiol Biotecnol. 2004; 10:1-15.

13. Justo MB, Guzmán GA, Mejia EG, Díaz CLG, Martínez $G$, Corona $E B$ (1998) Composición química de tres cepas mexicanas de setas Pleurotusostreatus. Arch Latinoam Nutr. 1998; 48(4):359-63.

14. Bonatti M, Karnopp P, Soares HM, Furlan SA. Evaluation of Pleurotus ostreatus y Pleurotus sajor-cajur nutricional characteristics when cultivated in differents lignocellulosic 
waste. Food Chem 2004; 88:125-428.

15. Roncero-Ramos Irene. Centro Tecnológico de Investigacion del Chapiñon de la rioja (CETICH) ed. Propiedades Nutricionales y saludables de los hongos, 2015, p 12-23.

16. Soobrattee MA, Neergheen VS, Luximon-Ramma A, Aruoma OI and Bahorun T. Phenolics as potential antioxidant therapeutic agents: mechanism and actions. Mutat. Res 2005; 579:200-13.

17. Rao AV and Rao LG. Carotenoids and human health. Pharmacol Res. 2007; 55: 207-16.

18. Robaszkiewicz A, Bartosz G, Lawrynowicz M and Soszynski. The role of Polyphenols, $\beta$-carotene and Lycopene in the antioxidative action of the extracts of dried edible mushrooms. J Nutr Metab. 2010; 2010:1-9.

19. Biesalski HK. Polyphenols and inflammation: basic interactions. Current Opinion Clin Nut Metab Care 2007; 10:724-8.

20. Markus MA and Morris BJ. Resveratrol in prevention and treatment of common clinical conditions of aging. Clin Interv Aging 2008; 3:331-9.

21. Lambert JD, Hong J, Yang Y, Liao J and Yang CS. Inhibition of carcinogenesis by polyphenol: evidence from laboratory investigations. Am J Clin Nutr. 2005; 81:284-91.

22. Romero L. Ayala N. Los hongos comestibles: una delicia gastronómica. Cátedra nacional de Biología "Juan Luis Cifuentes Lemus". Biología de la Conservación III. Uso, Manejo de la Biodiversidad. Universidad Autónoma del Estado de Hidalgo. Hidalgo, México, 2009.

23. Caballero Moreno A. Palacios Remondo J. Flora micológica de La Rioja. Zubía 1999; 17:11-43.

24. Bernas E. Jaworska G. Lisiewska Z. Edible mushroom as a resource of valuable nutritive constituens. Acta Sci, Pol, Technol Aliment. 2006, 5:5-20.

25. Wani B.A. Bonha R.H. Wani A.H. Nutritional and medicinal importance of mushrooms. J Med Plants Res, 2010, 4(24):2598-604.

26. Hernández-Rico G. y Moreno-Fuentes A. Hongos comestibles del género Amanita en el mercado de Acaxochitlán Hidalgo, México. Etnobiología 2010; 8:31-8.

27. Instituto Politecnico Nacional. Catálogo de hongos comestibles de Santiago Papasquiaro. Sistema de Administación de Programas y Proyectos de Invetigación, IPN ed., 2002, p 1-27.

28. Pardavé-Díaz LM. Contribución al conocimiento del género Amanita en el estado de Aguascalientes, México. Investigación y Ciencia 2001; 25:11-6.

29. García-Jimenez J. Singer $R$, Estrada $E$, Garza-Ocañas $F$, Valenzuela $R$. Dos especies nuevas del género Boletus (Boletales: Agaricomycetes) en México. Rev Mexicana Biodiversidad 2013; 84:152-62.

30. Bessette $A E$, Roody WC and Bessette AR. North American Boletes: A Color Guide to the Fleshy Pored Mushrooms. Syracuse University Press, Syracuse, NY 1-400, 2000.

31. Kosanic M, Rancovic B, Dasic M. Mushroom as possible antioxidant and antimicrobial agents. Iranian J Pharmaceut Res. 2012; 11(4):1095-102.

32. Hernández-Ayala M. Efectos de extractos orgánicos de Lactarius indigo sobre la viabilidad de líneas tumorales humanas. Tesis de Licenciatura. Universidad Michoacana de San Nicolás de Hidalgo. Michoacán, México. 2009.

33. Huerta G, Martínez-Carrera D, Sánchez JE, Leal-Lara $H$, Vilgalys $R$. Genetic relationships between mexican species of Pleurotus analyzing ITS-regions from rDNA. Micol Aplicada Internac. 2010; 22(1):15-25.

34. Rodríguez-Macías R. Caracterización de cepas del hongos comestible Pleurotus spp. en medios de cultivo y su evaluación en sustratos lignocelulósicos forrajeros para la producción de carpóforos. Tesis de maestría. Universidad Autónoma de Nuevo León. Nuevo León, México. 1996.

35. VarneroTM, Quiroz SM, Alvarez CH. Utilización de residuos forestales lignocelulósicos para producción del hongo ostra. Information Tecnológica 2010; 21(2):13-20.

36. Barros L, Venturini BA, Baptista P, Estevinho LM, and Ferreira ICFR. Chemical Composition and Biological Properties of Portuguese Wild Mushroom: A comprehensive Study. J Agric. Food Chem. 2008; 56: 3856-62.

37. Nouhra ER, Horton TR, Cazares E, Castellano M. Morphological and molecular Characterization of selected Ramaria Mycorrhizae. Mycorrhiza 2005; 15:55-9.

38. Cohen R. Persky L. Hadar Y. Biotechnological applications and potential of wood-degrading mushroom of the genus Pleurotus. Applied Microbiol Biotechnol. 2002; 8:37-45. 